\title{
Single-Period Balancing of Pay-Per-Click and Pay-Per-View Online Display Advertisements
}

\author{
Changhyun Kwon \\ Department of Industrial and Systems Engineering \\ University at Buffalo, the State University of New York
}

\begin{abstract}
In this paper, we study a balancing problem of web publishers for pay-per-view and pay-per-click contracts for online display advertising. Considering details of contracts, we refine prior research results on the recommendation of optimal strategies. We examine the problem by formalizing a simple stochastic optimization problem for a single period of advertising contracts. We investigate how pricing and other contract components will affect the optimal display strategies analytically and numerically.
\end{abstract}

\section{INTRODUCTION}

Online advertisements has become one of the most preferred advertising media in the last decade and the market has continuously grown. According to Interactive Advertising Bureau (2008), the Internet advertising revenue is total $\$ 23.4$ billion in the United States in 2008 , which is increased by 10.6 percent over 2007 . Among several other types of online advertising, there are two lead formats: search and display. When we type in keywords in a search engine, it returns the search results with relevant advertisements on the screen. On the other hand, when we visit a content providing web site, there are display advertisements in the formats of banner, rich media, digital video, etc. It is reported that search advertisements account for 45 percent ( $\$ 10.5$ billion) of 2008 full year revenues and display advertisements account for 34 percent ( $\$ 7.1$ billion).

For pricing online advertisements, the traditional 'pay-per-view' (PPV) has been dominant. In a PPV campaign, the advertiser pays the publisher proportional to the number of the advertisement viewed by population. If the advertisement is viewed once, its impressions is counted by one. After the campaign has finished, the total cost is determined by the cost-per-impression (CPM) times the number of impressions. Another popular pricing method is performance-based. One important measure of performance is the number of clicks, and the click-based pricing is called 'pay-per-click' (PPC). For a PPC advertisement, the advertiser pays the publisher the cost-per-click (CPC) times the number of clicks. If other actions are used to measure the performance, for example, purchasing, submission of forms, etc., the total cost is determined by the cost-per-action (CPA) times the number of actions. In 2008, Interactive Advertising Bureau (2008) reported 39 percent was priced by CPM, 57 percent by performance, and the rest by hybrid schemes.

In this paper, we are interested in a revenue maximization problem for display advertisements with two types of pricing contracts, PPV and PPC. In particular, we determines the optimal number of displays for PPC advertisements considering contracted impressions and penalty for PPV advertisements. As the number of page-views (capacity) is unknown a priori, we assume it is a random variable. We consider a single period capacity control problem to maximizing the revenue by balancing PPV and PPC advertisements.

Recently Geraghty (2008) investigated the synergy between revenue management and online advertising. However, there are only a few number of models have been reported in the perspective of revenue maximizing optimal strategies, although there are abundant literature in both separate areas. Mangani (2004) addresses optimal decision making between PPV and PPC advertisements when the publishers are price-takers in the display advertisement market and later Fjell (2009) revisited the same problem. Kumar and Sethi (2009) consider a dynamic pricing problem considering subscription and advertising and Roles and Fridgeirsdottir (2009) study a dynamic optimal customer selection and display scheduling problem for online display adver- 
tising when PPV is the only option. Fridgeirsdottir and Najafi Asadolahi (2009a) and Fridgeirsdottir and Najafi Asadolahi (2009b) also study revenue management problems with focusing advertising networks.

In Section 2, we present the stochastic optimization model for the single-period problem. In Section 3, We analyze the model and draw some insights in the same line with the previous research. We study the problem numerically in Section 4 and conclude this paper in Section 5.

\section{THE MODEL}

We consider one PPV advertisement and one PPC advertisement for a single period of advertising. For the period of advertising, suppose the publisher has already a contract of PPV advertisement for $v$ number of impressions to display. For the PPV contract, the advertiser pays by the number of impressions of the advertisement campaign. While, in practice, the contract is usually based on thousands of impressions, we consider for the simplicity pricing based on impressions. We use $p$ to denote the cost-per-impression (CPM).

While the publisher promised the PPV advertisement to display $v$ times, the number of page-view of the publishing web pages can be not enough. The publisher cannot know the exact number of page-view, hence, it is a random variable and we denote by $X$. We assume the publisher has the distribution information from historical data and proper forecasting. Let $F(\cdot)$ and $f(\cdot)$ denote the cumulative distribution function (cdf) and probability density function (pdf) of $X$, respectively. If $X$ turns out smaller than $v$, then there are unfulfilled impressions which incurs penalty cost for the publisher. We assume the penalty cost per unfulfilled impression is $h>0$. If $X$ turns out larger than $v$, so there are remaining capacity for displaying advertisements, the publisher can always display advertisements from advertising networks (network advertisements, for the rest of the paper) at a lower rate (Roles and Fridgeirsdottir, 2009). There is no obligation to guarantee the number of impressions for such advertisements. We denote the CPM for such advertisements by $q$ and we assume $p>q>0$.

Suppose the publisher receives a request of PPC advertisement campaign, while the publisher already has the PPV contract on hand. For the PPC advertisement, the publisher determines the number of impressions and the advertiser pays the cost based on the number of clicks. The click-through-rate (CTR), denoted by $C$, is unknown a priori, hence a random variable. We assume the CTR follows $\operatorname{cdf} G(\cdot)$ and pdf $g(\cdot)$. Let $\pi$ denote the cost-per-click (CPC), then the marginal revenue of displaying the PPC advertisement is determined by $\pi C$. If we display the PPC advertisement $u$ times, then the total revenue becomes $\pi C u$. However, because there is no promised number of impressions (no upper limit and lower limit), the advertiser may have to pay too much when the CTR is very high. To protect the advertiser, the PPC contract often states the budget limit for the total cost. If $\pi C u$ exceeds the budget limit, the publisher stops displaying the PPC advertisements and uses the remaining page-views to display the PPV advertisements or the network advertisements. Let $L$ be the budget limit.

Now the question is how many times the publisher should display the PPC advertisement to maximize profit. With two random variables $X$ and $C$, the expected profit as a function of $u$ is

$$
R(u)=p E[\min (X-u, v)]-h E[\max (u+v-X, 0)]+[\max (X-u-v, 0)]+E[\min (\pi C u, L)]
$$

where each term represents 'PPV revenue', 'PPV penalty', 'network advertisements revenue', and 'PPC revenue', respectively. This problem has a very similar structure to the famous newsvendor problem (see, for example, Birge and Louveaux, 1997; Porteus, 2002). While the demand is uncertain in the newsvendor problem, the supply (page-views) is uncertain and a few other components are added in (1). We can easily 
expand $R(u)$ to

$$
\begin{aligned}
R(u) & =p \int_{0}^{u+v} x f(x) d x-p u F(u+v)+p v(1-F(u+v)) \\
& -h(u+v) F(u+v)+h \int_{0}^{u+v} x f(x) d x \\
& +q\left(E[X]-\int_{0}^{u+v} x f(x) d x\right)-q(u+v)(1-F(u+v)) \\
& +\pi u \int_{0}^{L / \pi u} c g(c) d c+L\left(1-G\left(\frac{L}{\pi u}\right)\right)
\end{aligned}
$$

The first-order derivative is easily obtained as

$$
\frac{\partial R}{\partial u}=-p F(u+v)-h F(u+v)-q(1-F(u+v))+\pi \int_{0}^{L / \pi u} c g(c) d c
$$

and the second-order derivative is

$$
\frac{\partial^{2} R}{\partial u^{2}}=(q-p-h) f(u+v)-\frac{L^{2}}{\pi u^{3}} g\left(\frac{L}{\pi u}\right)
$$

From the assumption $p>q$, it is easy to see

$$
\frac{\partial^{2} R}{\partial u^{2}} \leq 0
$$

for all $u>0$, hence we have the following results.

Lemma 1 The revenue function $R(\cdot)$ is a concave function.

Lemma 2 The marginal revenue function $\frac{\partial R(\cdot)}{\partial u}$ is a non-increasing function.

Letting $\frac{\partial R}{\partial u}=0$, we obtain the condition for $\bar{u}$ to be optimal as following:

$$
(p+h-q) F(\bar{u}+v)=\pi \int_{0}^{L / \pi \bar{u}} c g(c) d c-q
$$

Note that (3) is only well-defined for $u>0$. Suppose the marginal revenue of the PPC advertisement is zero at $u \rightarrow 0$ is no greater than zero, i.e.,

$$
\lim _{u \rightarrow 0} \frac{\partial R(u)}{\partial u} \leq 0
$$

Then the marginal revenue of the PPC advertisement is never positive for all $u>0$ by Lemma 2 . Hence it is optimal to display no PPC advertisements. On the other hands, if

$$
\lim _{u \rightarrow 0} \frac{\partial R(u)}{\partial u}>0
$$

there exists a solution $\bar{u}>0$.

Theorem 3 The optimal number of impressions for the PPC advertisements is

$$
\begin{array}{ll}
u^{*}=0, & \text { if } \lim _{u \rightarrow 0} \frac{\partial R(u)}{\partial u} \leq 0 \\
u^{*}=\bar{u}, & \text { otherwise }
\end{array}
$$

where

$$
\lim _{u \rightarrow 0} \frac{\partial R(u)}{\partial u}=-p F(v)-h F(v)-q(1-F(v))+\pi E[C]
$$


and $\bar{u}$ is the solution of (3).

It is worth noting a special case when there is no limit on the CPC budget. If $L \rightarrow \infty$, we have

$$
\lim _{L \rightarrow \infty} \int_{0}^{L / \pi u} c g(c) d c=E[C]
$$

for all $0<u<\infty$. Then, the optimality condition (3) becomes

$$
(p+h-q) F\left(\bar{u}_{\infty}+v\right)=\pi E[C]-q
$$

where the solution is denoted by $\bar{u}_{\infty}$. Similar to Theorem 3 , we have the following result for the no budget limit case.

Corollary 4 When there is no budget limit for the PPC advertisement campaign, the optimal number of impressions for the PPC advertisements is

$$
\begin{array}{ll}
u_{\infty}^{*}=0, & \text { if } \frac{\partial R(0)}{\partial u} \leq 0 \\
u_{\infty}^{*}=\bar{u}, & \text { otherwise }
\end{array}
$$

where

$$
\frac{\partial R(0)}{\partial u}=-p F(v)-h F(v)-q(1-F(v))+\pi E[C]
$$

and

$$
\bar{u}_{\infty}=F^{-1}\left(\frac{\pi E[C]-q}{p+h-q}\right)-v
$$

The analytical solution form obtained in (6) and the other results in this section will lead to a few insights for the optimal strategies.

\section{SOLUTION PROPERTIES}

In this section, we identify the characteristics of the solution obtained in the previous section. First we investigate the case with no budget limit as in Corollary 4. We note that the inverse cdf of the page-view, $F^{-1}(\cdot)$, is well-defined in the interval $[0,1]$. From $(6)$, we observe that the following condition must be enforced

$$
0 \leq \frac{\pi E[C]-q}{p+h-q} \leq 1
$$

We characterize the solution when this condition is violated or tight, when there is no budget limit for the PPC advertisements.

Lemma 5 When $L \rightarrow \infty$, we have

$$
\pi E[C] \leq q \Longrightarrow u_{\infty}^{*}=0
$$

Proof. From (5), we have

$$
\begin{aligned}
\frac{\partial R(0)}{\partial u} & =-(p+h-q) F(v)-q+\pi E[C] \\
& \leq 0
\end{aligned}
$$

when $\pi E[C] \leq q$. Therefore, by Corollary 4 , we have $u_{\infty}^{*}=0$.

This results says: if the marginal revenue of the network advertisements exceeds the expected marginal revenue of displaying the PPC advertisements, it is never optimal to display the CPC advertisements. It will be better for the publisher to display the network advertisements if there is extra page-views. 
Lemma 6 When $L \rightarrow \infty$ and $0<F(v)<1$, we have

$$
\begin{aligned}
& \pi E[C]>p+h \Longrightarrow u_{\infty}^{*} \rightarrow \infty \\
& \pi E[C]=p+h \Longrightarrow u_{\infty}^{*}=F^{-1}(1)-v
\end{aligned}
$$

Proof. From (5) and the conditions $L \rightarrow \infty$ and $\pi E[C] \geq p+h$, we have

$$
\begin{aligned}
\frac{\partial R(0)}{\partial u} & =-(p+h-q) F(v)-q+\pi E[C] \\
& \geq-(p+h-q) F(v)-q+p+h \\
& =(p+h-q)(1-F(v)) \\
& >0
\end{aligned}
$$

Therefore we know $u_{\infty}^{*}>0$. Similarly from (2), we have

$$
\begin{aligned}
\frac{\partial R}{\partial u} & =-(p+h-q) F(u+v)-q+\pi E[C] \\
& \geq(p+h-q)(1-F(u+v)) \\
& \geq 0
\end{aligned}
$$

for all $u \geq 0$.

If $\pi E[C]>p+h$, the inequality (7) strictly holds, hence the marginal revenue is always strictly positive, i.e.,

$$
\frac{\partial R}{\partial u}>0 .
$$

Therefore, it is optimal that $u_{\infty}^{*} \rightarrow \infty$.

If $\pi E[C]=p+h$, the optimality condition gives us $F\left(u_{\infty}^{*}+v\right)=1$ or

$$
u_{\infty}^{*}=F^{-1}(1)-v
$$

For many continuous distribution functions, we also have $F^{-1}(1) \rightarrow \infty$. Therefore, we can say the following: if the expected marginal revenue of displaying the PPC advertisements exceeds the sum of the marginal revenue and the penalty of the PPV advertisements, it is optimal to display only the PPC advertisements.

The following property is natural.

Lemma 7 For all $L>0$, we have

$$
u^{*} \leq u_{\infty}^{*}
$$

Proof. The inverse $\operatorname{cdf} F^{-1}(\cdot)$ is a monotonically non-decreasing function. Therefore, from (3) we have

$$
\begin{aligned}
\bar{u} & =F^{-1}\left(\frac{\pi \int_{0}^{L / \pi \bar{u}} c g(c) d c-q}{p+h-q}\right)-v \\
& \leq F^{-1}\left(\frac{\pi E[C]-q}{p+h-q}\right)-v \\
& =\bar{u}_{\infty}
\end{aligned}
$$

since

$$
\int_{0}^{L / \pi \bar{u}} c g(c) d c \leq \int_{0}^{\infty} c g(c) d c \leq E[C]
$$

for all $\bar{u}>\infty$. Hence $u^{*} \leq u_{\infty}^{*}$.

From Lemmas 5 and 7 , we have the following. 
Lemma 8 When $0<L<\infty$, we have

$$
\pi E[C] \leq q \Longrightarrow u^{*}=0
$$

As in the no budget case, the same argument can be made for the finite budget case. If the marginal revenue of the network advertisements exceeds the expected marginal revenue of displaying the PPC advertisements, it is never optimal to display the PPC advertisements. However, there is little evidence to say the same argument in Lemma 6 for the finite budget case. We numerically observe a different behavior in Section 4, later in this paper.

The results in this section are somewhat different than the previous results reported in the literature. In particular, Mangani (2004) first raised the strategy for PPV and PPC advertisements when the publisher is a price taker in the market, and Fjell (2009) revisited the same topic, but arrived at a different conclusion. Mangani (2004) concludes that the publisher should consider a combination of two types of contracts. Later Fjell (2009) refined this result to that when the CTR is constant, only the PPV is chosen if the CTR is less than the ratio of PPV to PPC prices $(\pi C<p$ in our notation) and only the PPC is chosen if $\pi C>p$. However, in this paper, we have already noticed that this result can be further refined by considering the details of contracts. We summarize our findings in the same line of Mangani (2004) and Fjell (2009) as following:

Remark 9 Suppose $L \rightarrow \infty, F^{-1}(1) \rightarrow \infty$ and $0<F(v)<1$, then the following holds:

1. if the CTR is less than or equal to the ratio of the network ads price to CPC, the publisher should always choose PPV only; and

2. if the CTR is greater than the ratio of the sum of $C P M$ and PPV penalty to $C P C$, the publisher should always choose PPC only.

We can provide further managerial insight from Remark 9. If the CTR is not too low (opposite to Case 1), the publisher could generate more revenue by a combination of PPV and PPC contracts. In most cases, the promised number of displays is smaller than the expected number of page-views (otherwise, the publisher would face large amount of penalty cost by not displaying advertisements). Therefore, by mixing PPC contracts, the publisher could avoid losing revenue-generating opportunity. If the CTR is not too high (opposite to Case 2), the publisher should also consider a mix of both advertisement types, instead of displaying only PPC advertisements. That is, by the mix, the publisher could protect himself from low $\mathrm{CTR}$, and in consequence low revenue from PPC contract.

\section{NUMERICAL STUDY AND STRATEGY UPDATE}

In this section, we provide a computational scheme for finding $u^{*}$ when there is a finite budget limit for the PPC advertisements. When there is no limit $(L \rightarrow \infty)$, the solution is given by $u_{\infty}^{*}$ in Corollary 4 . Therefore we only consider $L$ is finite. We know, from Theorem 3, when $\frac{\partial R(0)}{\partial u} \leq 0$, we have the solution $u^{*}=0$. Hence we are only interested in computing $u^{*}$ when $\frac{\partial R(0)}{\partial u}>0$. We are seeking $u^{*}>0$ that maximizes $R(\cdot)$. Because $R(\cdot)$ is a concave function, we may consider any type of line search method for solving. See Bazaraa et al. (2006) for such methods. We give a statement of the Bolzano's bisection search method, the most simplest search method, as following.

\section{$\underline{\text { Bisection Search Method }}$}

Step 0 Let $\left[a_{0}, b_{0}\right]$ be an interval such that $\frac{\partial R\left(a_{0}\right)}{\partial u}>0$ and $\frac{\partial R\left(b_{0}\right)}{\partial u}<0$, and set $k=0$.

Step 1 Let $u_{k}=\frac{1}{2}\left(a_{k}+b_{k}\right)$.

- If $\frac{\partial R\left(u_{k}\right)}{\partial u}<0$, set $\left[a_{k+1}, b_{k+1}\right]=\left[a_{k}, u_{k}\right]$.

- If $\frac{\partial R\left(u_{k}\right)}{\partial u}>0$, set $\left[a_{k+1}, b_{k+1}\right]=\left[u_{k}, b_{k}\right]$. 


\begin{tabular}{ll|ll}
\hline$p$ & 1.00 & $X$ & $\sim N\left(3500,800^{2}\right)$ \\
$h$ & 0.50 & $C$ & $\sim N\left(0.1,0.01^{2}\right)$ \\
$q$ & 0.10 & $\pi$ & 10.00 \\
$v$ & 3000 & & \\
\hline
\end{tabular}

Table 1: Parameter Values

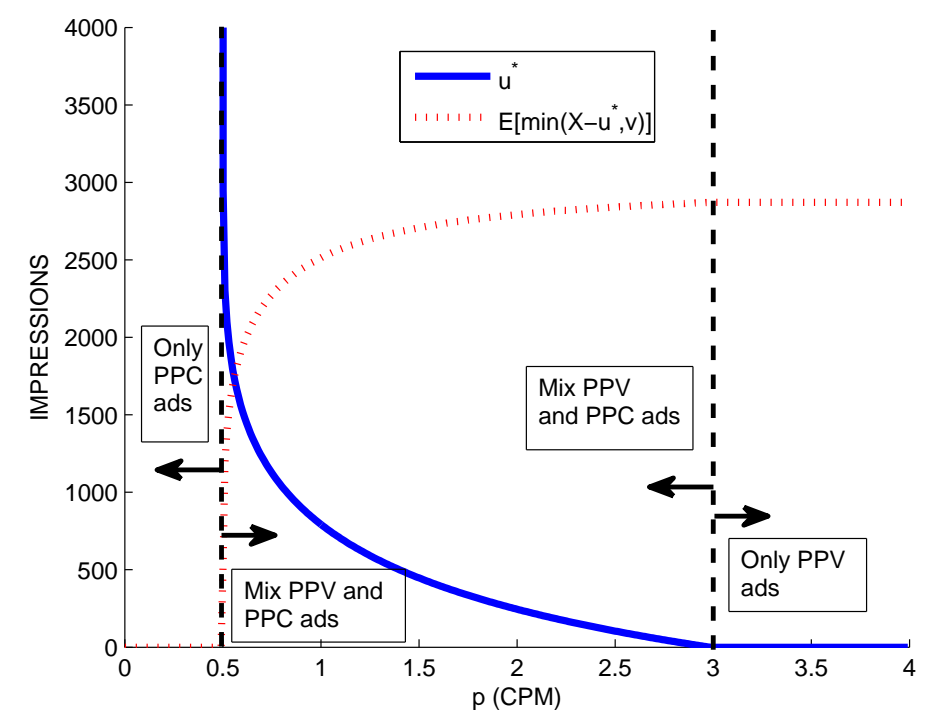

Figure 1: Optimal stratagies vs. CPM, when there is no budget limit

Step 2 If $\left|b_{k+1}-a_{k+1}\right|<\varepsilon$ for a preset tolerance $\varepsilon>0$, stop and declare

$$
u^{*} \approx u_{k+1}=\frac{a_{k+1}+b_{k+1}}{2}
$$

Otherwise, set $k=k+1$ and go to Step 1 .

For each iteration, we need to evaluation $F\left(u_{k}+v\right)$ and $\int_{0}^{L / \pi u_{k}} c g(c) d c$. For the most of popular probabilistic distributions, $F\left(u_{k}+v\right)$ can be computed by closed-form expressions and $\int_{0}^{L / \pi u_{k}} c g(c) d c$ can be approximated by using various numerical integration techniques, for example, the trapezoidal rule. See Stoer and Bulirsch (2002).

Using the computational scheme, we study the behavior of the optimum when the parameter values are given in Table 4. We assumed Normal distributions for both page-views and CTR. First we consider there is no budget limit $(L \rightarrow \infty)$. While fixing the other parameters as in Table 4 , we change the CPM, $p$. The result is shown in Figure 1. Similarly, we change the CPC, $\pi$, and the result is shown in Figure 2. The dotted curves represent the expected number of impressions of the PPV advertisements when we employ $u^{*}$, i.e., $E\left[\min \left(X-u^{*}, v\right)\right]$. As investigated in Remark 9, we again confirm that there exists the zone of prices when we need to consider a mix of PPV and PPC advertisements for maximizing the expected revenue.

When there is a finite budget for the PPC advertisements, for example $L=5,000$, we observe quite different optimalities. As seen in Figure 3, the behavior of the optimal values is exactly same when we change $p(\mathrm{CPM})$ and keep $\pi=10.00(\mathrm{CPC})$. However, when we change $\pi(\mathrm{CPM})$, the behavior is very different than no budget limit case, as presented in Figure 4. We observe the same behavior of $u^{*}$ until $\pi$ reaches the point A. After passing the point A, $u^{*}$ starts to decrease. Due to the budget limit, when $\pi$ is high, the publisher need not to consider displaying the PPC advertisements more. Instead, it is optimal for the publisher to display more the PPV advertisements to maximize the expected revenue. When there is a 


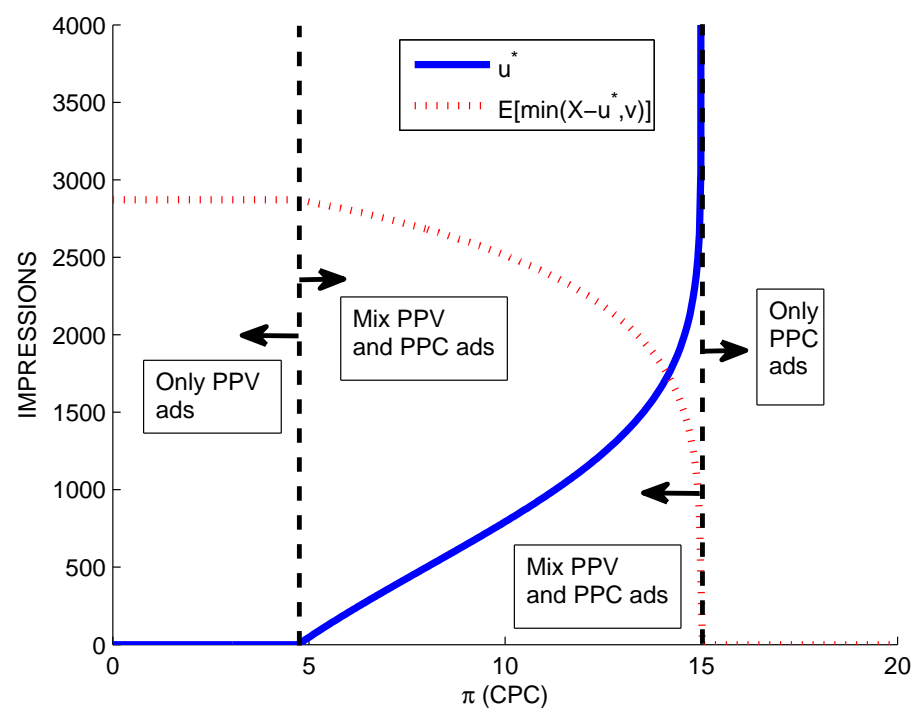

Figure 2: Optimal strategies vs. CPC, when there is no budget limit

budget limit, the publisher will display only PPV advertisements when the CPC is very low, and again will display mostly PPV advertisements when the CPC is very high, i.e., if $\pi \rightarrow \infty$, then $u^{*} \rightarrow 0$.

We note that the optimal $u^{*}$ can be computed when the information on $v, X$ and $C$ are given along with the other parameters. Let $u^{*}=u^{*}(v, X, C)$ where the random variables $X$ and $C$ as arguments mean we use the corresponding distribution functions as input. While exercising the optimal strategy $u^{*}$, we may find either our previous estimation and forecasting on $X$ and $C$ very different than the reality, or there is excess page-views after displaying the PPC advertisements all of $u^{*}(v, X, C)$ times. Then we are required to update our optimal strategy. Suppose we have displayed the PPV advertisements $v_{1}$ times so far and we have the updated information on the page-view and the CTR as $X^{\prime}$ and $C^{\prime}$. Then we can update our optimal strategy for the rest of time period by

$$
u^{* \prime}=u^{*}\left(v-v_{1}, X^{\prime}, C^{\prime}\right)
$$

We note that the updated strategy is irrelevant to how many times we have displayed the PPC advertisements so far.

\section{CONCLUSION}

For the relatively new advertising media, the Internet, there are many problems remaining unsolved. Among them, in particular, there has been a debate on how to balance the two popular online advertising contracts: the traditional PPV and the performance-based PPC. We have looked at the problem by formalizing and analyzing a simple stochastic optimization problem. We assumed the publisher holds a PPV contracts with a promised number of impressions and receives a request of PPC contract. We provided the optimal strategy to determine the number of impressions to display the PPC advertisements.

We confirm the previous research that the publisher has to consider only one of them in the extreme ends, for example, CPM or CPC is too high or too low. On the other hand, we have arrived yet another conclusion by considering the details of contracts: there exists a zone of prices where we have to consider a mix of both contracts. In particular, the findings of this paper are:

1. Regardless of the budget limit for the CPC contracts, if the expected CTR is less than or equal to the ratio of the advertising network advertisements price to the $\mathrm{CPC}$, the publisher should always choose displaying the PPV advertisements only. 


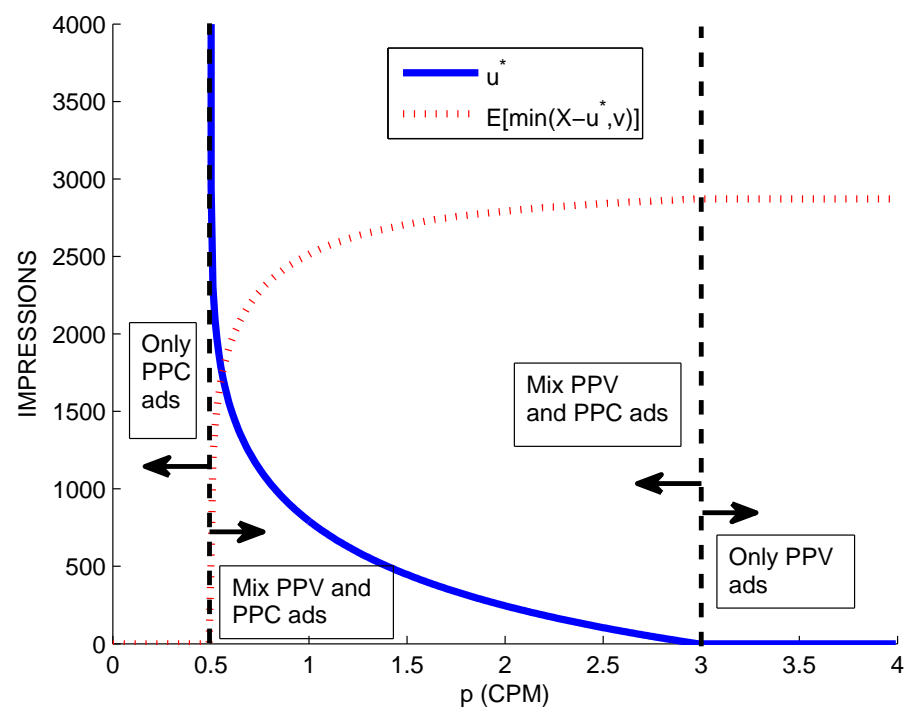

Figure 3: Optimal strategies vs. CPM, when there is a finite budget limit $(\mathrm{L}=5,000)$

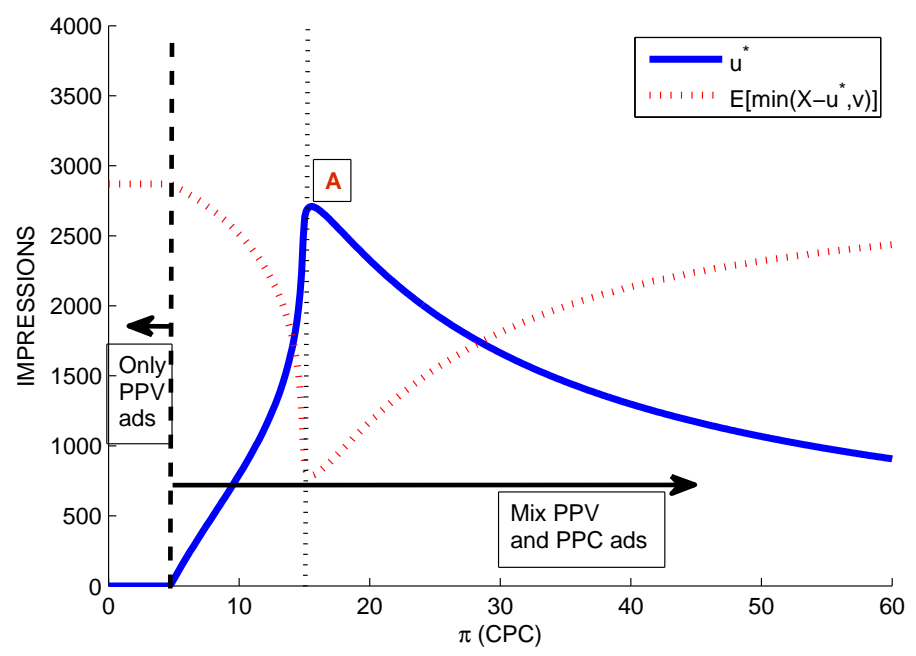

Figure 4: Optimal strategies vs. $\mathrm{CPC}$, when there is a finite budget limit $(\mathrm{L}=5,000)$ 
2. When there is no budget limit for the CPC contracts, if the expected CTR is greater than the ratio of the sum of CPM and PPV penalty to CPC, the publisher should always choose displaying the PPC advertisements only.

3. When there is a finite budget limit for the CPC contracts, even when the CPC is very high, it can be possible that the publisher should consider displaying mostly the PPV advertisements.

Note that, although we have given some discussions how many times of display of each advertisement is optimal, we have provided no operational guideline for the order in which the publisher display the mix of the PPV and PPC advertisements. A microscopic investigation must be followed to provide the scheduling of display. In addition, we have only considered one PPV contract and one PPC contract for a single period. It is left for the future research to explore the optimal strategies that balance two contracts for multiple PPV and PPC contracts for multiple periods.

\section{References}

Bazaraa, M. S., H. D. Sherali, and C. M. Shetty (2006). Nonlinear Programming: Theory and Algorithms (3rd ed.). Wiley-Interscience.

Birge, J. and F. Louveaux (1997). Introduction to stochastic programming. Springer.

Fjell, K. (2009). Online advertising: Pay-per-view versus pay-per-click - A comment. Journal of Revenue and Pricing Management 8, 200-206.

Fridgeirsdottir, K. and S. Najafi Asadolahi (2009a). Revenue management for online advertising using advertising networks. Working Paper.

Fridgeirsdottir, K. and S. Najafi Asadolahi (2009b). The Optimal Pay-Per-Click Pricing of Display Advertising in Advertising Networks: A Queuing Systems Approach. Working Paper.

Geraghty, K. (2008). Revenue Management \& Digital Marketing. Analytics Winter, 8-13.

Interactive Advertising Bureau (2008). IAB Internet Advertising Revenue Report. http://www.iab.net/insights_research/530422/1357.

Kumar, S. and S. Sethi (2009). Dynamic pricing and advertising for web content providers. European Journal of Operational Research 197(3), 924-944.

Mangani, A. (2004). Online advertising: Pay-per-view versus pay-per-click. Journal of Revenue and Pricing Management 2(4), 295-302.

Porteus, E. (2002). Foundations of stochastic inventory theory. Stanford University Press.

Roles, G. and K. Fridgeirsdottir (2009). Dynamic Revenue Management for Online Display Advertising. Journal of Revenue and Pricing Management forthcoming.

Stoer, J. and R. Bulirsch (2002). Introduction to Numerical Analysis. Springer. 